\title{
Some considerations on the position control of pneumatic cylinders
}

\author{
Yukio KAWAKAMI \\ Shibaura Institute of Technology, 307 Fukasaku Omiya Saitama 330, JAPAN \\ Yoshihiko MASUDA, Sunao KAWAI \\ Waseda University, 3-4-1 Okubo Shinjuku Tokyo 169, JAPAN
}

\begin{abstract}
The difficulty of the precise positioning by the pneumatic servo system has not been clearly explicated. This paper analyzes the dynamic behavior of the pneumatic cylinder on the position control system and examines factors that make a precise positioning difficult. It is certain that the main factor is the complex relation between the friction force and the compressibility of the air, which causes the time lag in the driving force. From these results, the effective steps to realize the precise positioning with the pneumatic cylinder are described. Practically, it is possible to compensate the influence of this time lag and improve the position control features by means of the use of the pressure cascade control loop. It is also proposed that the pressure cascade control can be replaced with the acceleration feedback control which has the more simple composition.
\end{abstract}

\section{KEYWORDS}

Pneumatic cylinder, Position control, Friction, Pressure feedback, Acceleration feedback

\section{INTRODUCTION}

The demand for automation and energy saving in various fields of industry has brought about an increase in the usage of pneumatic equipments. Pneumatic actuators are economical, easy to operate and can be driven with enough driving force at high speed, and therefore, they are widely used as operating units in various machines. However, now there are two important technical problems about pneumatic actuators. One is high-speed driving of the pneumatic actuators in order to enhance productivity. The other is precise and speedy positioning with the pneumatic servo systems in order to realize automatic machines of good quality.

In earlier papers ${ }^{(1)-(2)}$, the authors have studied the former problem, high-speed driving of the pneumatic cylinders. Then it was found out that the pressure response of the air in the cylinder chamber becomes oscillatory and that the velocity response also shows an oscillatory behavior when a pneumatic cylinder is driven at high-speed. From this result, the authors have proposed a new method for high-speed driving of the pneumatic cylinder. The method consists of the effective application of such oscillatory velocity responses, which can reduce impact force at the end of the stroke without actively controlling valve opening area. The authors are now studying about the pneumatic servo system as a next problem.

The purpose of this paper is to investigate the precise and speedy position control system with the pneumatic cylinder to realize a more convenient pneumatic servo system. Firstly it is examined what factor makes precise position control difficult. This examination indicates that the friction force at the load

Fluid Power. Edited by T. Maeda. (C) 1993 E \& FN Spon. ISBN 0419191003. 
has enough value to interfere with the driving force, and that the compressibility of the air brings about a time lag in the pressure response of the air. So it is important that the influence of the friction force and of the compressibility of the air is compensated. From these results, the effective steps are described in order to realize the precise and speedy positioning with the pneumatic cylinder. Practically, it is possible to compensate the influence of the compressibility of the air and improve the position control features according to the use of the pressure cascade control loop. It is also proposed that the pressure cascade control can be replaced with the acceleration feedback control which has the more simply composition.

\section{EXPERIMENTAL APPARATUS OF PNEUMATIC POSITIONING SYSTEM}

A schematic diagram in Fig. 1 shows the experimental apparatus of the positioning system with the pneumatic cylinder which is used in this study. The pneumatic cylinder in this system has the diameter of the piston $50[\mathrm{~mm}]$, the stroke $600[\mathrm{~mm}]$, and can drive load mass about $100[\mathrm{~kg}]$. The friction force acting on the pneumatic cylinder is not so large because the load was moved with linear bearings. The measurements of the friction force for the pneumatic cylinder are shown in Fig.2, and they are used in the simulation model. The controller is composed of a microprocessor and interfaces which input or output signals. The signal of the piston displacement is measured by the potentiometer, and this signal is converted into the approximate velocity and acceleration signals through the OP-amp circuits.

Experimental result of the step response is shown in Fig. 3 when the position feedback control with the only displacement signal is practiced in this system. In the small loop gain, two kinds of oscillatory behavior

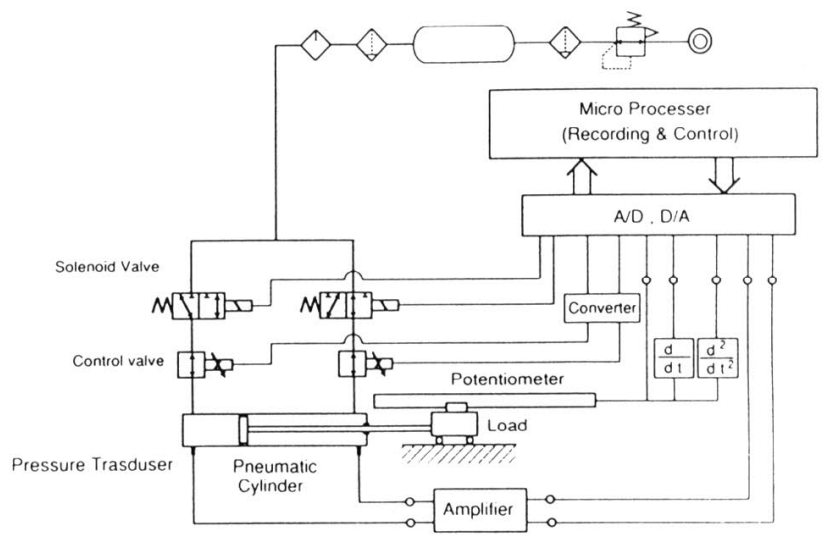

Fig.1 A schematic diagram of the experimental apparatus

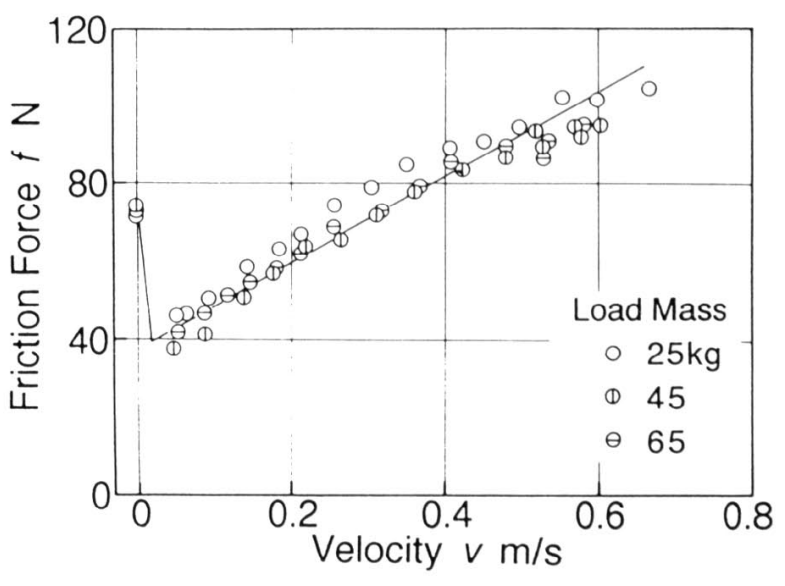

Fig.2 Experimental results of the friction force

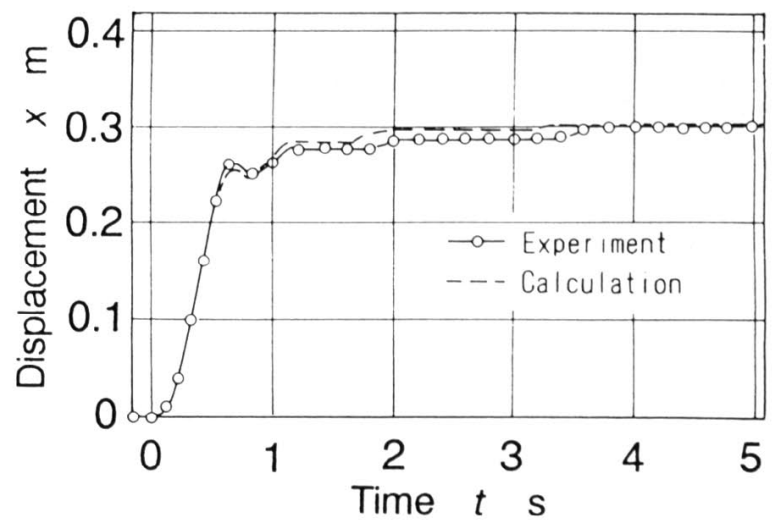

Fig.3 Experimental results of the step response

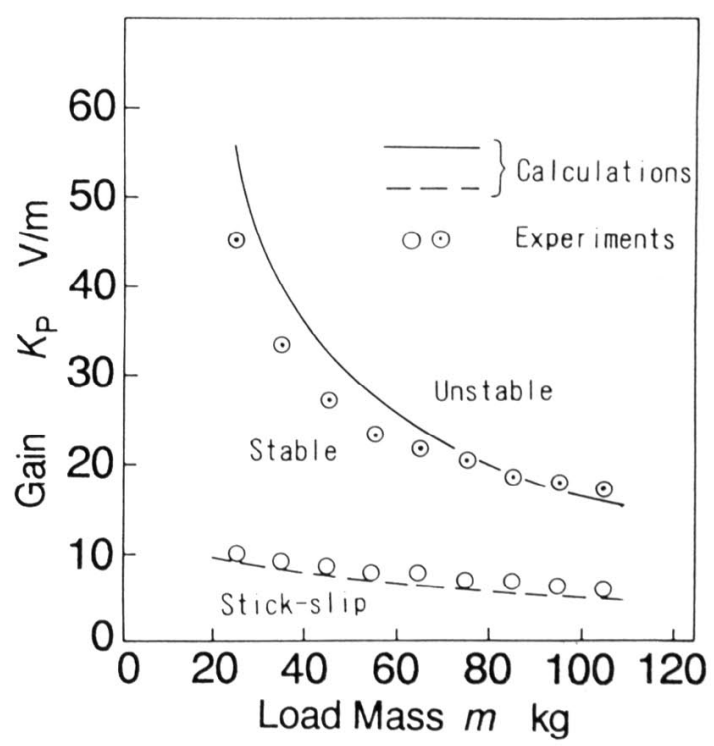

Fig.4 Stability limit of the pneumatic positioning system 
takes place. Before the piston reaches near the reference point, the responses of displacement are oscillatory without overshooting. This oscillation is caused by the compressibility of the air. Near the reference point, the piston stops and moves frequently. This behavior is generally known as the stick-slip phenomenon caused by the friction. However, in the pneumatic system the mechanism that the oscillatory behavior takes place is more complex. It is detailed later.

Fig. 4 shows the stability of this experimental system. The vertical axis means the loop gain in the position feedback control and the horizontal axis means the load mass. The stability area is between upper limited gain (solid line) and lower limited gain (broken line). Then solid line and dotted plots mean typical maximum stability gain on the system, and broken line and normal plots mean the limit that stick-slip occurs. So it is difficult to apply the appropriate loop gain for precise positioning in the pneumatic system.

By the way, results calculated with the simulation model ${ }^{(3)}$ are compared with experimental results in Fig. 3 \& 4. It is found that the calculated results are nearly equal to the experimental results and the validity of the simulation model be confirmed. Therefore this simulation model will be used for analyzing the dynamic behavior of the pneumatic cylinder system.

\section{EXAMINATION OF RESPONSES NEAR THE REFERENCE POINT}

In the pneumatic system, the loop gain is generally set for small value because the air has a time lag caused by the compressibility. However, from Fig 3, it is found that the piston with the positioning system of the pneumatic cylinder stops and moves frequently near the reference point in a small loop gain. It will be examined how this oscillatory behavior occurs.

Fig. 5 shows calculated results in the small loop gain and details transit of the driving force near the reference point with the enlarged view. From this figure it is found that the friction force has enough value to interfere with the driving force near the reference point, because that the compressibility of the air brings about a time lag in the air pressure response. So the piston stops for a little while when the velocity of the piston is equal to zero near the reference point and the driving force is inferior to the static friction force (A-point). Then value of the driving force gradually grows up as the air flows into cylinder chamber. When the driving force gets superior to the static friction force, the piston begin moving again (B-point). After that, it is difficult to make the piston stop on the reference point because of a time lag in the system (C-point). And this cycle (from A to C) repeats without setting the piston on the reference point.

Therefore, it is important that the influence of the friction force and of the compressibility of the air is compensated.
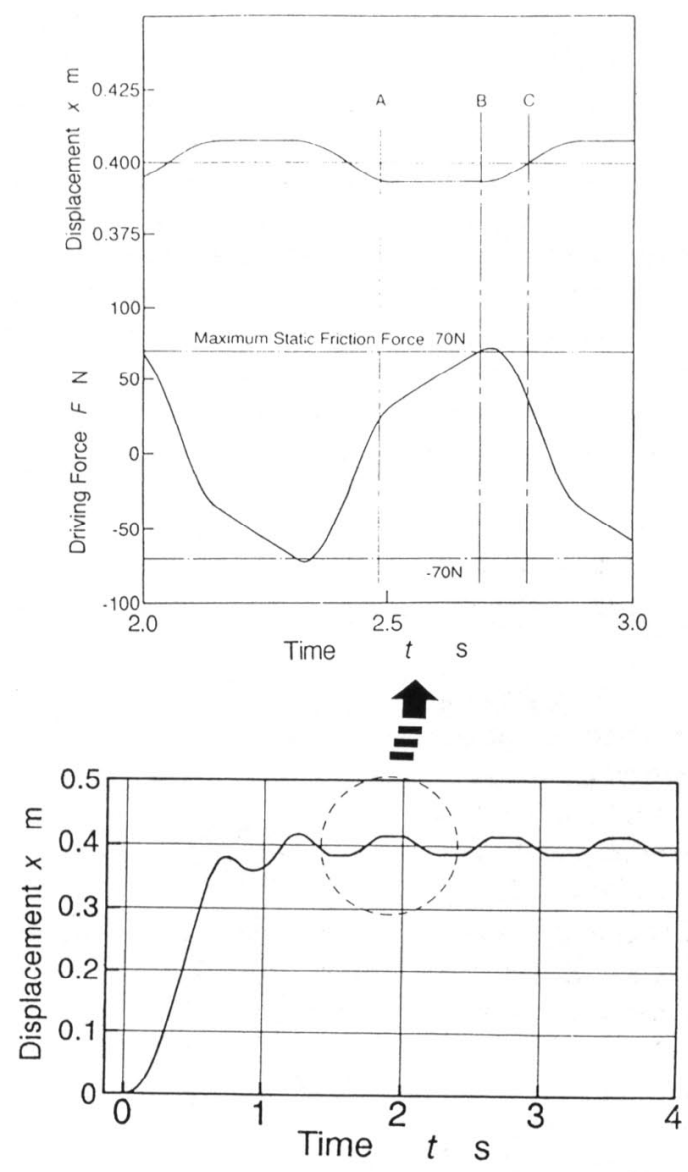

Fig.5 The oscillatory behavior near the reference point

\section{ON ACCURACY OF THE PNEUMATIC POSITIONING SYSTEMS}

\section{INFLUENCE OF THE FRICTION}

As mentioned before, the static friction is one of main factors which causes the oscillatory behavior near the reference point. However, the viscous friction contributes to an effect of damping on pneumatic systems. Fig 6 shows the calculated responses of the pneumatic cylinder system driving the load, if the system has no friction and control valves are in full open. It is found that the oscillatory behavior caused by the compressibility of the air takes place and the stability of the system gets worse. So the effective feedback loop of the velocity is necessary for the positioning system of good quality when the friction has a little effect of a damping on the system.

Next, Fig. 7 investigates the influence of the static friction. The solid line means the calculated response where the static friction force is very small. It is apparent from this figure, a precise positioning without oscillatory behavior near the reference point is realized when the static friction is in a negligible degree and the 


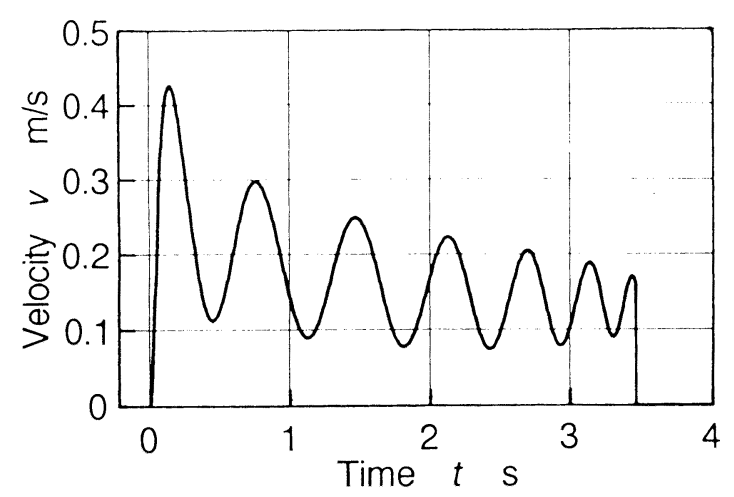

Fig.6 Calculated response with no friction force

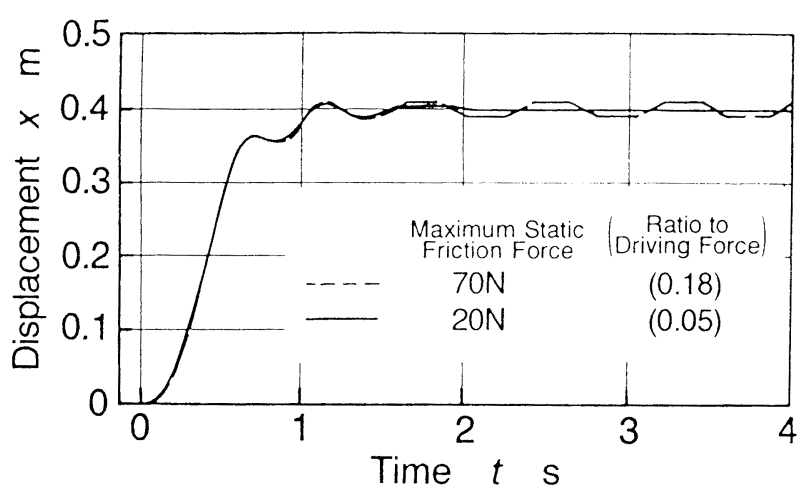

Fig. 7 Influence of the static friction

system has the viscous friction as a damping. However, the static friction is not small in general conditions for use. Therefore, it is important to consider the method for a precise positioning in the pneumatic system with the static friction.

\section{RELATION WITH MAXIMUM DRIVING FORCE AND STATIC FRICTION FORCE}

As mentioned in Fig.5, the compressibility of the air brings about a time lag in the driving force with the pneumatic positioning system. In comparison with the unique behavior of the driving force in the pneumatic system, Fig. 8 shows calculated responses with the electric positioning system. It has the same static friction as the experimental system has in this study. From this figure, it is found that the friction force doesn't interfere with the driving force and the positioning of good quality is realized in the electric system because the driving force has no time lag for the control signal from the regulator.

In the pneumatic system, it is explained that the complex relation between the friction force and the compressibility of the air make the positioning difficult.

And the ratio of the maximum static friction force to the maximum driving force is an important factor, because

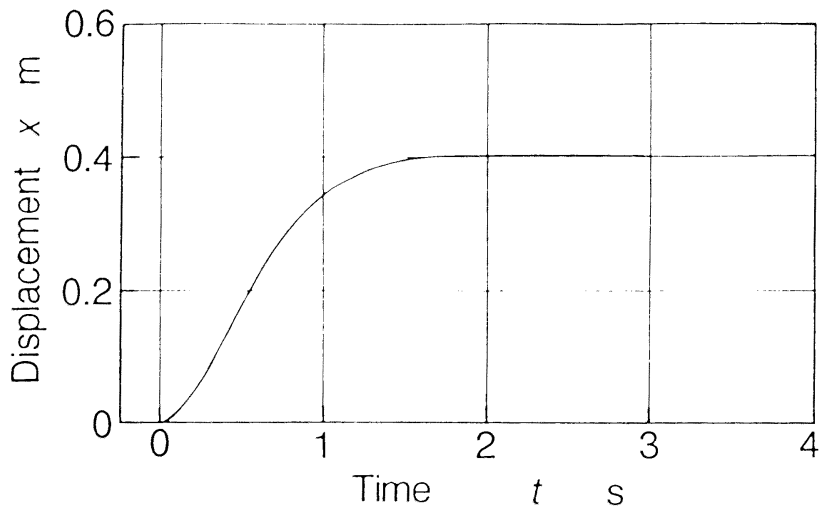

Fig.8 The step response with electric positioning system

value of the ratio has a large influence on qualities of the positioning in the pneumatic system. Practically, the precise positioning in the pneumatic system can be achieved when the ratio is small enough not to interfere with the driving force. So it is necessary that the driving force is sufficiently large in the pneumatic system. (In the experimental system, the ratio is approximately 0.2 , which is relatively high.) The following figure investigates the ratio from the point in the design of the pneumatic positioning system.

Fig. 9 \& 10 show calculated responses when the ratio is regarded as a parameter. In Fig. 9 the cylinder diameter is made alternated, while in Fig. 10 the supply pressure is made alternated. From these figures, it is clearly that a precise positioning is achieved in the system with a fairy small ratio. However, Fig.9 \& 10 are not the best way in the pneumatic systems. In Fig.9, the time lag caused by a cylinder capacity is bigger, when the cylinder diameter is larger. In Fig. 10, the ratio is not small enough, because the maximum supply pressure is limited by pneumatic equipments. Consequently, it is difficult that the driving force is sufficiently large in the pneumatic system. The following investigates the other way to achieve the precise positioning in the pneumatic system.

\section{IMPROVEMENT OF THE POSITIONING PERFORMANCE}

As mentioned before, the factors which make the precise positioning difficult are that the friction force at the load has enough value to interfere with the driving force, and that the compressibility of the air brings about a time lag in the pressure response of the air. Then it is investigated that the factors are compensated to improve the performance of the pneumatic positioning system.

Fig.5 explains that the piston frequently stops and moves near the reference point because the driving force has the time las caused by the complex relation between the friction force and the compressibility of the air: And the time lag decides the performance of the 


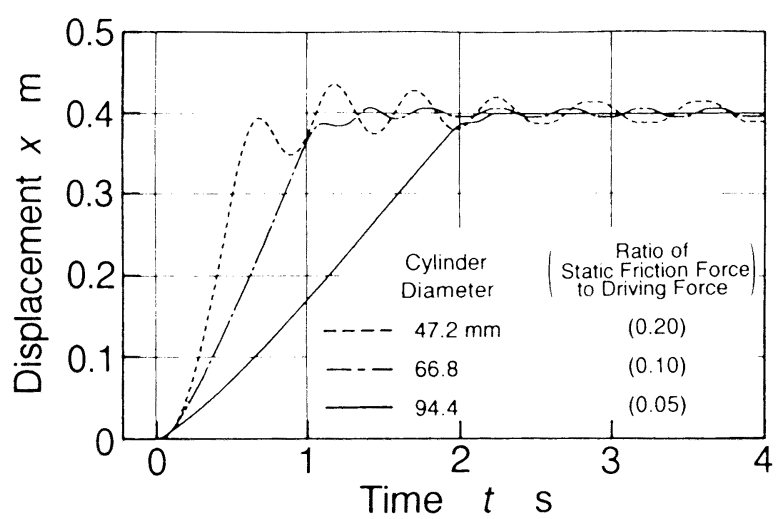

Fig.9 Influence of the ratio of the friction force to maximum driving force (The cylinder diameter is variable)

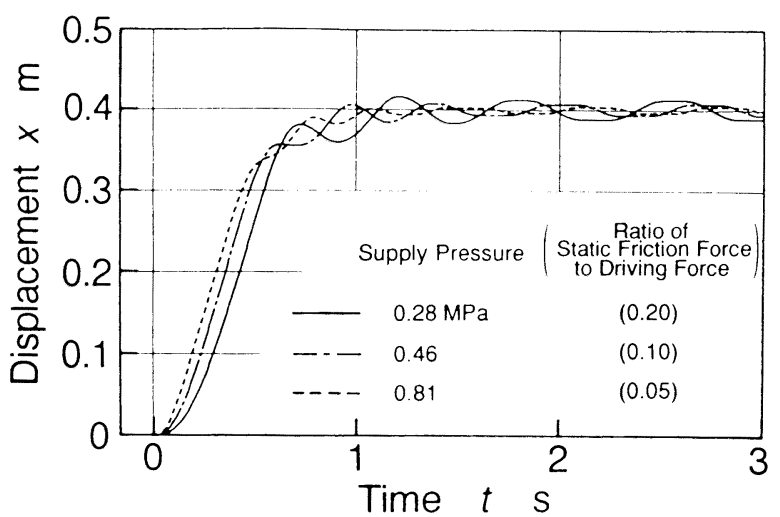

Fig.10 Influence of the ratio of the friction force to maximum driving force (The supply pressure is variable)

positioning in the pneumatic system. Therefore, it is investigated that a pressure cascade feedback loop is appended to the pneumatic positioning system to compensate the time lag. Fig. 11 shows a block diagram of the pneumatic positioning system with the pressure feedback loop. And Fig. 12 shows a calculated result of the step response in the system. From this figure, it is found that the performance of the positioning is improved by the compensation of the time lag with the pressure feedback loop. In some previous papers ${ }^{(4)-(5)}$ about the pneumatic positioning system, the pressure feedback loop is applied, but it is not explained why the pressure feedback loop is effective to improved the performance of the positioning.

Additionally, it is clearly that the pressure cascade feedback loop is almost equal to the acceleration feedback loop from a block diagram in Fig. 13. So the authors propose that the acceleration feedback loop should be applied to the positioning system in order to achieve the precise positioning in the pneumatic system. The following investigates the acceleration feedback loop.

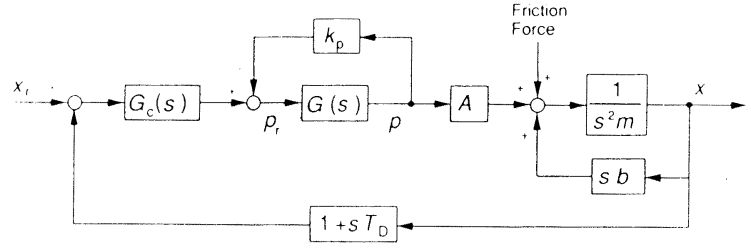

Fig.11 A block diagram with the pressure cascade feedback loop

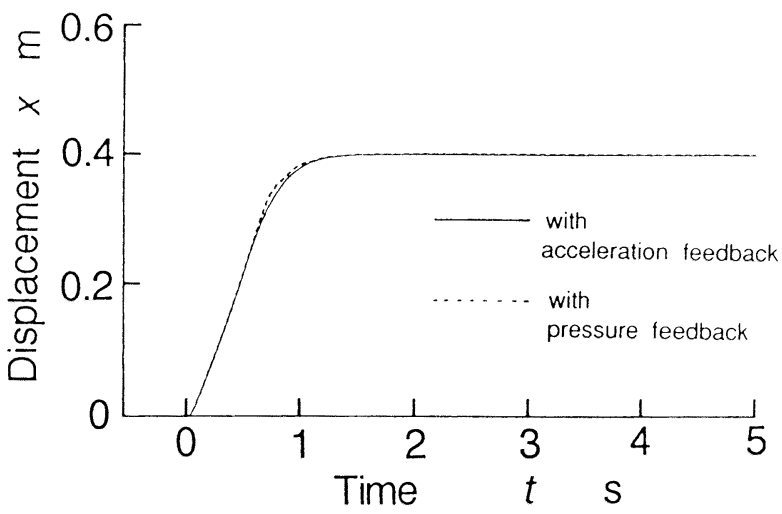

Fig.12 Effects on the pressure cascade feedback and the acceleration feedback

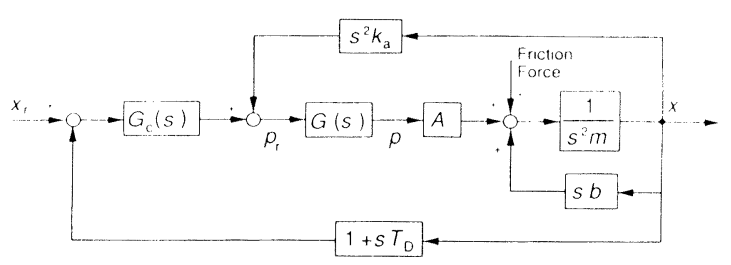

Fig.13 A block diagram with the acceleration feedback loop

\section{EFFECTS OF THE ACCELERATION FEEDBACK LOOP}

From Fig. $11 \&$ 13, it is necessary that the loop gain of the acceleration feedback is set for value as is equivalent to that of the pressure cascade feedback. However, the influence of the friction force on the acceleration feedback loop is different from that on the pressure feedback loop, because the friction force is regarded as a disturbance. So both responses are compared in order to examine the difference. Fig. 12 also shows a calculated result of the step response in the system with the acceleration feedback loop and that with the pressure feedback loop. From this figure, it is found that both responses are almost the same and the acceleration feedback loop and the pressure feedback loop compensate the time lag of the driving force. 

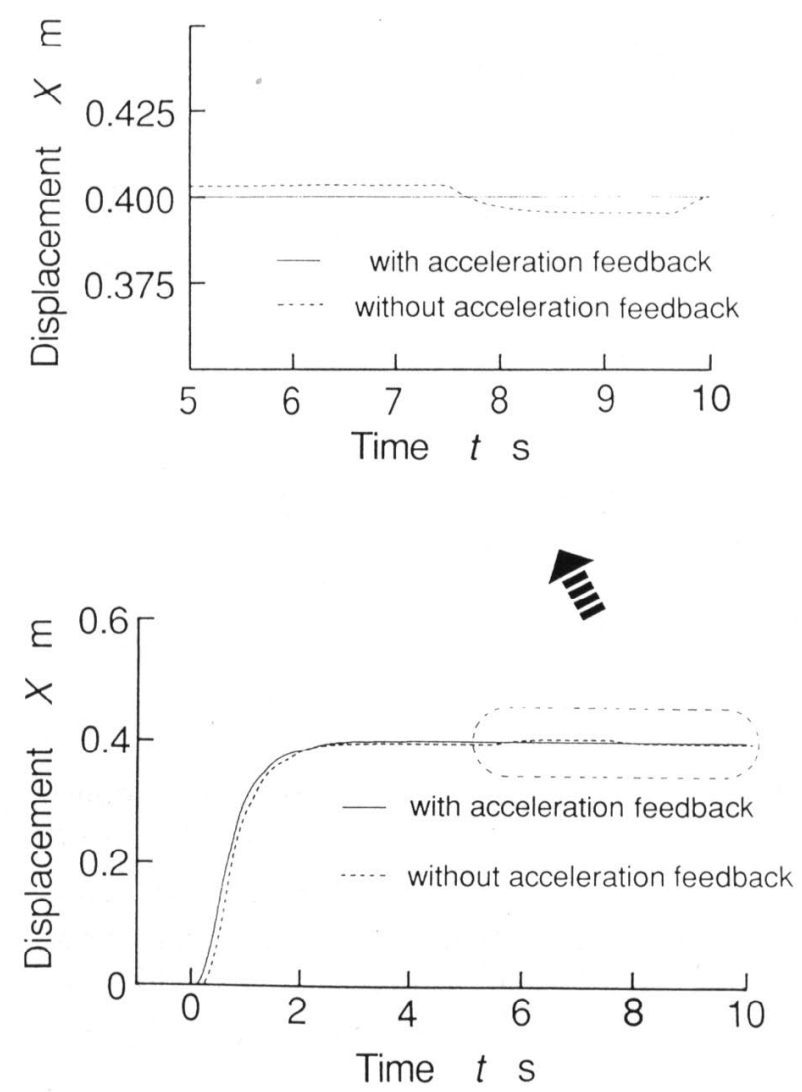

Fig.14 Experimental results with the acceleration feedback loop

Therefore, this examination proves the validity that the loop gain of the acceleration feedback is set for value equivalent to that of the pressure cascade feedback. Practically the loop gain should be set for value in which the time lag of the driving force is much smaller than that of the load.

Additionally, the effect of the acceleration feedback loop is verified in experiments. In the experimental apparatus of Fig. 1, it is into an approximate signal of the acceleration that the OP-amp circuit converts a measured signal of displacement. Fig.14 shows an experimental result of the step response with the acceleration feedback loop. It is apparent from this figure, a precise positioning without oscillatory behavior near the reference point is achieved with the acceleration feedback loop.

\section{CONCLUSION}

This paper analyzes the dynamic behavior on the pneumatic cylinder for the positioning system and investigates the problems to realize the precise pneumatic positioning. The summarized results are as follows.

1. In the small loop gain, the response near the reference point has an oscillatory behavior, which caused by the complex relation between the friction force and the compressibility of the air. This behavior is a main factor which makes the pneumatic positioning difficult.

2 . The ratio of the maximum static friction force to the maximum driving force has a large influence on the performance of the positioning in the pneumatic system. Practically, the precise positioning in the pneumatic system can be achieved when the ratio is small enough not to interfere with the driving force.

3. In the positioning system with the pressure cascade feedback loop, the performance of the positioning is improved by the compensation of the time lag caused by the compressibility of the air. But the structure of this system is complicated.

4. It is certain that the pressure cascade feedback loop is almost equal to the acceleration feedback loop. So the loop gain of the acceleration feedback should be set for value equivalent to that of the pressure cascade feedback. 5 . The authors propose that the acceleration feedback loop should be applied to the positioning system in order to achieve the precise positioning in the pneumatic system. And the appropriateness of this loop can be verified by experiments.

\section{ACKNOWLEDGMENTS}

The authors would like to thank Professor Tadahiro MACHIYAMA of Department of Mechanical Engineering, Nippon Institute of Technology for his interest and continuous support.

\section{REFERENCES}

1. Kawakami,Y., Kimura,S., Kawai,S. and Machiyama, T., Some considerations on the oscillatory behavior of pneumatic cylinders, Proceedings of First JHPS International Symposium on Fluid power, 1989, $251 / 258$

2. Kawakami, Y., Takeda, R. and Kawai, S., Some considerations on the generalized conditions for highspeed driving of pneumatic cylinders, Proceedings of Flucome' $91,1991,543 / 548$

3. Kawakami, Y., Akao, J., Kawai, S. and Machiyama, T., Some considerations on the dynamic characteristics of pneumatic cylinders, Journal of Fluid control, 1988, 19-2, 22/36

4. Kawamura, S., Miyata, K., Hanafusa, H. and Ishida, K., A hierarchical feedback control scheme for pneumatic drive system, Trans. of SICE, 1990, 26-2, $204 / 210$

5. Noritugu, T., Wada. T., Control performance and typical features of pneumatic servo system, Journal of JHPS, 1990, 21-4, 417/424 